\title{
Dissecting cognitive stages with time-resolved fMRI data: a comparison of fuzzy clustering and independent component analysis
}

Citation for published version (APA):

Smolders, A., de Martino, F., Staeren, N. P. M. C., Scheunders, P., Sijbers, J., Goebel, R. W., \& Formisano, E. (2007). Dissecting cognitive stages with time-resolved fMRI data: a comparison of fuzzy clustering and independent component analysis. Magnetic Resonance Imaging, 25(6), 860-8. https://doi.org/10.1016/j.mri.2007.02.018

Document status and date:

Published: 01/01/2007

DOI:

10.1016/j.mri.2007.02.018

Document Version:

Publisher's PDF, also known as Version of record

\section{Document license:}

Taverne

Please check the document version of this publication:

- A submitted manuscript is the version of the article upon submission and before peer-review. There can be important differences between the submitted version and the official published version of record.

People interested in the research are advised to contact the author for the final version of the publication, or visit the DOI to the publisher's website.

- The final author version and the galley proof are versions of the publication after peer review.

- The final published version features the final layout of the paper including the volume, issue and page numbers.

Link to publication

\footnotetext{
General rights rights.

- You may freely distribute the URL identifying the publication in the public portal. please follow below link for the End User Agreement:

www.umlib.nl/taverne-license

Take down policy

If you believe that this document breaches copyright please contact us at:

repository@maastrichtuniversity.nl

providing details and we will investigate your claim.
}

Copyright and moral rights for the publications made accessible in the public portal are retained by the authors and/or other copyright owners and it is a condition of accessing publications that users recognise and abide by the legal requirements associated with these

- Users may download and print one copy of any publication from the public portal for the purpose of private study or research.

- You may not further distribute the material or use it for any profit-making activity or commercial gain

If the publication is distributed under the terms of Article $25 \mathrm{fa}$ of the Dutch Copyright Act, indicated by the "Taverne" license above, 


\title{
Dissecting cognitive stages with time-resolved fMRI data: a comparison of fuzzy clustering and independent component analysis
}

\author{
Alain Smolders ${ }^{\mathrm{a}}$, Federico De Martino ${ }^{\mathrm{b}}$, Noël Staeren ${ }^{\mathrm{b}}$, Paul Scheunders ${ }^{\mathrm{b}}$, \\ Jan Sijbers ${ }^{\mathrm{b}}$, Rainer Goebel ${ }^{\mathrm{b}}$, Elia Formisano ${ }^{\mathrm{b}, *}$ \\ ${ }^{\mathrm{a}}$ Vision Lab (Department of Physics), University of Antwerp, Universiteitsplein 1, B-2610 Antwerpen, Belgium \\ ${ }^{\mathrm{b}}$ Department of Cognitive Neuroscience, Faculty of Psychology, Maastricht University, 6200 MD Maastricht, The Netherlands \\ Accepted 11 January 2007
}

\begin{abstract}
In combination with cognitive tasks entailing sequences of sensory and cognitive processes, event-related acquisition schemes allow using functional MRI to examine not only the topography but also the temporal sequence of cortical activation across brain regions (time-resolved fMRI). In this study, we compared two data-driven methods - fuzzy clustering method (FCM) and independent component analysis (ICA) - in the context of time-resolved fMRI data collected during the performance of a newly devised visual imagery task. We analyzed a multisubject fMRI data set using both methods and compared their results in terms of within- and between-subject consistency and spatial and temporal correspondence of obtained maps and time courses. Both FCM and spatial ICA allowed discriminating the contribution of distinct networks of brain regions to the main cognitive stages of the task (auditory perception, mental imagery and behavioural response), with good agreement across methods. Whereas ICA worked optimally on the original time series, averaging with respect to the task onset (and thus introducing some a priori information on the stimulation protocol) was found to be indispensable in the case of FCM. On averaged time series, FCM led to a richer decomposition of the spatio-temporal patterns of activation and allowed a finer separation of the neurocognitive processes subserving the mental imagery task.

This study confirms the efficacy of the two examined methods in the data-driven estimation of hemodynamic responses in time-resolved fMRI studies and provides empirical guidelines to their use.
\end{abstract}

(C) 2007 Elsevier Inc. All rights reserved.

Keywords: fMRI; Fuzzy clustering; Independent component analysis; Data-driven analysis; Mental imagery

\section{Introduction}

Since its introduction in 1992 [1], functional magnetic resonance imaging (fMRI) is used widely to identify the spatial layout of brain activation associated with sensory stimulations, motor actions and cognitive tasks [2]. The recent combination of event-related acquisition schemes and methods for accurate estimation of the blood oxygenation level dependent (BOLD) responses allows examining, in some cases, not only the topography but also the temporal sequence of cortical activation across brain regions [3,4]. The achievable temporal resolution is limited to a few

\footnotetext{
* Corresponding author. Department of Neurocognition, Faculty of Psychology, Maastricht University, 6200 MD Maastricht, The Netherlands. Tel.: +31 43 3884040; fax: +31 433884125 .

E-mail address: e.formisano@psychology.unimaas.nl (E. Formisano).
}

hundred milliseconds by the sluggishness and variability of the hemodynamic responses (HRs) [5]. This approach of time-resolved fMRI is thus useful particularly in the study of complex cognition, in combination with cognitive tasks entailing sequences of cognitive processes and relatively long neural processing times. A recent study, for example, used time-resolved fMRI to address the issue of functional differentiation between the various cortical regions subserving a complex task of visuo-spatial mental imagery [6].

In the analysis of time-resolved fMRI data, the accurate estimation of task-related BOLD responses assumes a particular relevance. Complex tasks normally involve the activation of extended networks of brain regions with widely different HRs. A region involved in the maintenance in memory of one or more items, for example, may exhibit a much more sustained neural (and hemodynamic) response than a region that transiently responds to the switching 
between two conditions. Additionally, within each region, a substantial degree of experimentally induced trial-by-trial variability is to be expected. In such cases, the analysis of HRs based on conventional model (or hypothesis) driven methods (such as the general linear model [7]) may not be optimal. Indeed, these methods assume a spatially invariant parametric model of the HR function and this may result in a different sensitivity in one or another region of the brain. Furthermore, they do not account for the trial-by-trial variability of the responses.

A complementary approach to estimate the spatiotemporal pattern of brain activation is to use data-driven methods, such as independent component analysis (ICA) [8] and clustering techniques [9]. In both these approaches, a new and potentially more informative representation of the data is obtained by decomposing the original time series into a set of spatio-temporal modes, without strong a priori assumptions about the temporal profile of the effects of interest.

As applied to fMRI time series analysis, ICA attempts to separate blindly data into a set of non-Gaussian and 'spatially' statistically independent modes (independent components or ICs, see below) [8]. The ability of spatial ICA to distinguish between neurophysiologically interesting sources and noise sources has been a matter of investigation in several recent publications $[10,11]$. Spatial ICA has been shown to outperform principal component analysis (PCA). One difficulty, however, consists in the fact that ICA does not provide any intrinsic order of the ICs. The experimenter is thus confronted with the problem of selecting and interpreting a subset of these components [12-14].

Clustering techniques separate time series into several patterns according to the similarity among them. A wellknown member of this category is the fuzzy clustering method (FCM) [15]. In the first FCM applications in neuroimaging, clustering was performed directly on the time series, using the Euclidean distance to quantify the similarity among the acquired signals $[15,16]$. To overcome problems related to noise, a distance measure was introduced based on the correlation between the HRF and a stimulus function, rather than the raw time series [17]. Other studies compared FCM to alternative techniques in the field of fMRI, like correlation [18] or PCA [19]. Several studies dealt with other aspects, like the cluster validity problem [20-22] or the influence of higher fields on FCM results [23]. Clustering on features extracted from the fMRI time series at each voxel was also investigated [24,25]. Typical drawbacks of clustering approaches are the need of an a priori definition of the number of clusters (cluster validity problem) and the negative influence on the algorithm's results caused by the large amount of inactivated voxels (illbalanced data problem). As in ICA, a post hoc interpretation of the resulting clusters is required.

As illustrated in Refs. [6,26], the new spatio-temporal representation of the data obtained by either clustering or spatial ICA has several appealing properties in the context of time-resolved fMRI. Firstly, the description of the sequence of spatial patterns of brain activation is obtained blindly, thus reducing the problem of having an explicit model of the HR. Secondly, each spatio-temporal mode includes voxels with co-varying time courses of activity and thus this representation is very helpful in highlighting the simultaneous involvement of spatially remote brain regions in the same stage of the task (functional connectivity). It is thus of interest to examine similarity and differences between the results obtained using these two different data-driven approaches. A comparison of spatial ICA and clustering has been recently described in Ref. [27]. However, this comparison was limited to a block-designed experiment with a simple visual stimulation.

In the present study, we investigate and compare the ability of FCM and ICA to estimate the spatio-temporal patterns of brain activity in the context of time-resolved fMRI measurements collected during the performance of a newly devised mental imagery task. This complex task requires the construction, maintenance and comparison to a visual target of simple geometric figures, mentally created from auditory instructions. We analyze a multisubject fMRI data set using both methods and compare their results in terms of withinand between-subject consistency and spatial and temporal correspondence of obtained maps and time courses.

\section{Methods}

\subsection{Fuzzy clustering}

Fuzzy clustering attempts to partition a subset of $N$ voxels in C 'clusters' of activation [28]. This is achieved by comparing the voxel's time courses $\mathbf{x}_{n}(n=1 \ldots N)$ with each other and assigning them to representative time courses, called cluster centroids $\mathbf{v}_{c}(c=1 \ldots C)$, derived during this process. Fuzziness relates to the fact that a voxel is generally not uniquely assigned to one cluster only (hard clustering), but instead, the similarity of the voxel time course to each cluster centroid is determined. This is expressed by the 'membership' $u_{c n}$ of voxel $n$ to cluster $c$. For each voxel, we have:

$\sum_{c=1}^{C} u_{c n}=1$

Both centroids $\mathbf{v}_{c}$ and memberships $u_{c n}$ are updated in an iterative procedure, elaborated by Bezdek et al. [29] and expressed by:

$\mathbf{v}_{c}=\frac{\sum_{n=1}^{N} \boldsymbol{u}_{c n}{ }^{m} \mathbf{x}_{n}}{\sum_{n=1}^{N} \boldsymbol{u}_{c n}{ }^{m}} \quad \boldsymbol{u}_{c n}=\frac{1}{\sum_{k=1}^{C}\left(\frac{\boldsymbol{d}\left(\mathbf{x}_{n}, \mathbf{v}_{c}\right)}{\boldsymbol{d}\left(\mathbf{x}_{n}, \mathbf{v}_{k}\right)}\right)^{\frac{2}{m-2}}}$

where $d$ is a distance measure, determining the similarity between the time course of a voxel and a cluster center, and $m$ is the fuzziness coefficient, determining the fuzziness of 
the procedure and used to 'tune out' the noise in the data. Theoretically, $m$ lies between 1 (smallest fuzziness) and infinity. Its ideal value, however, is problem dependent. Several distance measures $d$ can be defined: the Euclidean distance $d_{\mathrm{E}}$ and the Mahalanobis distance $d_{\mathrm{M}}$ [30] are mostly used and are defined as:

$d_{\mathrm{E}}\left(\mathbf{x}_{n}, \mathbf{v}_{c}\right)=\left\|\mathbf{x}_{n}-\mathbf{v}_{c}\right\|^{2}$

$d_{\mathrm{M}}\left(\mathbf{x}_{n}, \mathbf{v}_{c}\right)=\left(\mathbf{x}_{n}-\mathbf{v}_{c}\right)^{\mathrm{T}} \Sigma_{c}^{-1}\left(\mathbf{x}_{n}-\mathbf{v}_{c}\right)$

where $\sum_{c}$ represents the covariance matrix of cluster $c$. The Mahalanobis distance takes into account the actual (elliptical) shape of the cluster, i.e., instead of treating all voxels $\mathbf{x}_{n}$ equally when calculating the distance $d$ to the cluster centre $\mathbf{v}_{c}$, it weights the differences by the range of variability, described by $\sum_{c}$, in the direction of the voxel. The Euclidean distance does not take into account the shape of the cluster, i.e., it assumes a spherical shape, corresponding to a covariance matrix $\sum_{c}$ with $1 \mathrm{~s}$ on the main diagonal and 0 s elsewhere.

The algorithm starts from an initial set of membership values for the data set, expressed in matrix form as:

$U^{(0)}=\left(1-\frac{\sqrt{2}}{2}\right) U+\frac{\sqrt{2}}{2} V$

with $U=1 / C$ and $V$ a matrix of randomly chosen cluster centres. Next, the new cluster centres and memberships are computed using Eq. (2). The procedure terminates when successive iterations do not further change significantly memberships and cluster centres, as calculated by Eq. (2). This procedure corresponds to the minimization of the following objective function:

$\sigma_{\mathrm{w}}^{2}=\frac{1}{N} \sum_{n=1}^{N} \sum_{c=1}^{C} u_{c n}^{m} d\left(\mathbf{x}_{n}, \mathbf{v}_{c}\right)$

which computes the within-class variance over all clusters $\sigma_{\mathrm{w}}^{2}$. In practice, a user-defined threshold for change in $\sigma_{\mathrm{w}}^{2}$ determines when convergence is reached.

The a priori determination of the fuzziness coefficient and the number of clusters are research topics often encountered in the literature [20]. Although several heuristics are introduced, the result is often problem dependent.

Preprocessing includes the transformation of each time series into its $z$-score so as to avoid the clustering algorithm to classify the voxels based on signal amplitude, instead of signal shape. Finally, PCA is performed to reduce data dimensionality.

\subsection{Spatial ICA}

Let $X$ be the $T \times M$ matrix of the fMRI time series. Each row contains an fMRI image (the spatial processes) of $M$ voxels $(m=1 \ldots M)$ acquired at time $t(t=1 \ldots T)$, with $T$ the number of scans. Let $S$ be the $N \times M$ matrix whose rows $S_{n}(n=1 \ldots N)$ contain the independent images $(N \leq T)$ and $A$ the $T \times N$ mixing matrix whose columns $A_{n}$ contain the time courses of the $N$ independent images and is assumed to be of full rank.

The problem of the ICA decomposition of fMRI time series can be formulated as the estimation of both matrices of the right side of the following equation:

$X=A S$

under the constraint that the images $S_{n}$ are (in the ideal case) spatially independent. No a priori assumption is made about the mixing matrix $A$, i.e., about the time courses corresponding to the independent images. In this model, all the spatial components, with the possible exception of one, are assumed to be non-Gaussian. Structured (non-Gaussian) artifacts in the data (e.g., head movements, machine and physiological artifacts) are not explicitly modelled, but instead are treated as independent sources and are expected to be represented in one or more of the components. The amount of statistical dependence within a fixed number of spatial components can be quantified by means of their mutual information. Thus, the ICA decomposition of $X$ can be defined as a linear transformation:

$S=W X$

where the matrix $W$ (the 'unmixing' matrix) is determined such that the mutual information of the target components $S_{n}$ is minimized. Matrix $A$ can be computed as the pseudoinverse of $W$. Note that this definition of ICA and Eq. (8) imply that ICs are determined up to a permutation, a multiplicative constant and to the sign.

We estimated $S$ using cortex-based ICA (cb-ICA) [31] as implemented in BrainVoyagerQX (Brain Innovation, Maastricht, The Netherlands). Cb-ICA uses individual anatomical constraints and a fixed-point ICA algorithm (FastICA) [32] and allows an optimized analysis of cortical sources. After sphering the matrix $X$ and reduction of the temporal dimension of the data set with PCA, the hierarchical (deflation) mode of the FastICA algorithm was used and the components were estimated one by one. After the decomposition, voxel values of IC spatial maps were $z$-transformed and colour coded according to the absolute value and sign [8]. It should be noted that the $z$-scores do not pertain to any significance statistic, because no comparison is made to a null hypothesis.

\section{3. fMRI data}

Fuzzy clustering and spatial ICA were compared in the context of an fMRI investigation of visuo-spatial mental imagery. During the functional measurements (see below), subjects were asked to create a mental representation of simple two-dimensional geometric figures based on a sequence of auditory instructions sequentially building up each figure. After a jittered delay, subjects had to mentally rotate the internally constructed image, in accordance with a 
visually presented target figure, rotated over an angle $\left(40^{\circ}\right.$, $80^{\circ}$ or $120^{\circ}$ ), and had to indicate with a button press whether these were identical or mirror inversed. This task involves a sequence of sensory, cognitive and motor processes that can take up to several seconds and can thus be studied using fMRI mental chronometry.

Three healthy, young subjects participated to the experiment. For each subject, several successive fMRI data runs were recorded in a single session: three for subject 'LM', three for subject ' $\mathrm{AB}$ ' and four for subject ' $\mathrm{CJ}$ '. During each run, 18 trials were acquired, each trial lasting $30 \mathrm{~s}$. Functional scans consisted of 23 transversal slices acquired on a 3-T Siemens Allegra (Siemens, Erlangen, Germany) scanner using a single-shot gradient-echo echo-planar imaging sequence $(\mathrm{TE}=30 \mathrm{~ms}, \mathrm{TR}=1500 \mathrm{~ms}$, matrix size $=64 \times 64$, voxel size $=3.5 \times 3.5 \times 4 \mathrm{~mm}, 730$ images). During the same session each subject underwent a highresolution T1-weighted anatomical scan (MDEFT sequence, voxel size $=1 \times 1 \times 1 \mathrm{~mm}, 176$ slices per slab, slice thickness $=1 \mathrm{~mm}$, data matrix $=224 \times 256 \mathrm{~mm}, \mathrm{TR}=7.92 \mathrm{~s}$, $\mathrm{TE}=2.4 \mathrm{~ms}$ ).

\subsection{Preprocessing}

The fMRI time series were subject to a series of preprocessing steps using BrainVoyagerQX (Brain Innovation, Maastricht, The Netherlands):

(1) Slice scan time correction was performed by resampling the time courses with sinc interpolation such that all voxels in a given volume represent the signal at the same point in time.

(2) Head movements were corrected automatically minimizing the sum of squares of the voxel-wise intensity differences between each volume and the first volume of each run. Each volume was then resampled in three-dimensional space according to the optimal parameters using trilinear interpolation.

(3) Temporal high pass filtering was performed to remove temporal drifts of a frequency below five cycles per run.

(4) After co-registration to the anatomical images collected in the same session, functional volumes were projected into Talairach space [33].

(5) For each of the original 10 functional time series (three subjects, three runs for subject ' $A B$ ' and ' $\mathrm{LM}$ ' and four runs for subject ' $\mathrm{CJ}$ '), an averaged data set was obtained considering an interval of $30 \mathrm{~s}$ around the onset of each auditory instruction $(2 \mathrm{~s}$ prestimulus onset and $28 \mathrm{~s}$ poststimulus) corresponding to 20 time points. This was done to increase the signal-to-noise ratio of consistently task-related (CTR) processes. The effect of this averaging was evaluated for both methods (FCM and cb-ICA). In case of FCM, we found that averaging over the trials was indispensable to have a reasonable detection power of CTR processes. In case of ICA, we found that averaging over the trials deteriorates the results. Therefore, in this study, we consider FCM of averaged data and ICA of the original time series.

\subsection{Fuzzy clustering and spatial ICA: selection of parameters and visualization}

Both FCM and sICA require setting of specific parameters (fuzziness coefficient, number of clusters, number of ICs), which can have a substantial influence on the results of the analyses and the comparisons between methods. Here we determined these settings by a preliminary analysis and inspection of one functional time series (subject 'LM', Run 1). For this dataset, as for the other datasets (see Results and Discussion), maps obtained by FCM and spatial ICA were superimposed to three-dimensional anatomical images and/or projected on an inflated and flattened representation of the cortical sheet of the subject's brain. This latter representation allows displaying in one picture the spatial topography of the clusters/ICs, thus providing a useful tool for their interpretation.

To determine an appropriate value of the fuzziness coefficient $m$, we applied FCM for a range of values of $m$ between 1 and 3. After visual assessment of the topography and time course of the resulting clusters, 1.25 was chosen as an acceptable value for the fuzziness coefficient, which is in close agreement with literature [20]. The number of clusters was fixed to 13 for all the extractions. We applied PCA to the data sets under investigation and typically retained 13 of the 20 total dimensions, capturing at least $90 \%$ of its variance/covariance. Initialising to higher or lower number of clusters yield to nonoptimal results. In case of lower number of clusters, some 'meaningful' clusters (see below) were not found and in case of higher number of clusters they were split into several clusters. In order to determine the influence of the initialization step, during which membership values and cluster centres are randomly chosen, we repeated the FCM decomposition multiple times (a data set was analysed 100 times) and results were compared across multiple extractions. No significant difference in results was observed, indicating the robustness of the method to random initialization.

When using cb-ICA, a PCA-based reduction of dimensions was performed prior to the ICA decomposition. Based on data inspection the number of retained dimensions was fixed to 30 , accounting for more than $99 \%$ of the variance/ covariance of the data.

\subsection{Fuzzy clustering and spatial sICA: selection of clusters/ components and comparison}

To assess and compare the results of FCM and spatial ICA, we focused on the clusters/components whose representative time courses were clearly related to the imagery task. Clusters/components were compared, qualitatively, by visual inspections of the maps and time courses and, quantitatively, by calculating the spatial (temporal) 
cross-correlation coefficients between maps (time courses). Furthermore, for both FCM and spatial ICA we analyzed the consistency of the results across runs (within subjects) and subjects.

\section{Results and discussion}

\subsection{FCM and spatial ICA maps and time courses}

Figs. 1 and 2 show a representative example of maps and time courses obtained using FCM and spatial ICA. In Fig. 1, IC (A) and cluster (B) maps are colour coded and projected on a flattened reconstruction of the subject (LM, Run 1) cortex. Overlay of cluster maps corresponds to membership values in the range of 0.5 to 1 . Hence, only those voxels clearly assigned to a single cluster are shown. Overlay of IC maps corresponds to normalized amplitudes greater than 1.8. Cluster centre time courses (solid line) and eventrelated averaged IC time courses (dash-dot line) are illustrated in Fig. 2.

Both FCM and spatial ICA decompositions allowed highlighting the contribution of distinct networks of areas to the sequential stages of the task, with good agreement across methods. According to the sequential ordering of the representative time courses (see Fig. 2), a first cluster/IC (light green) included regions of the transverse temporal gyrus and of the superior temporal sulcus/gyrus bilaterally, reflecting the activation of the auditory cortex at the beginning of the trial. Interesting, in some FCM decompositions (see Table 1) this 'auditory' cluster was dissected into two clusters, whose time courses and spatial topography are compatible with a distinction between early auditory regions and language-specific regions (dark green). Following cluster(s)/IC(s) (light and dark blue) were representative of brain activation during the performance of the visuospatial mental imagery task. Two clusters and one/two ICs (see Table 1) were found to include bilateral frontal and posterior parietal regions which have been previously involved in similar tasks of mental imagery [6]. Regions of the occipito-temporal cortex were also included in these maps. The time courses of these two clusters/ICs suggested a sequential involvement of corresponding cortical networks in early and late stages of the imagery task. Finally, one or two cluster(s)/IC(s) (see Table 1) reflected the activity in the occipital (red) and in the occipito-parietal and motor regions (yellow), mostly dominated by response related to the visual presentation of the target stimulus and consequent motor response.

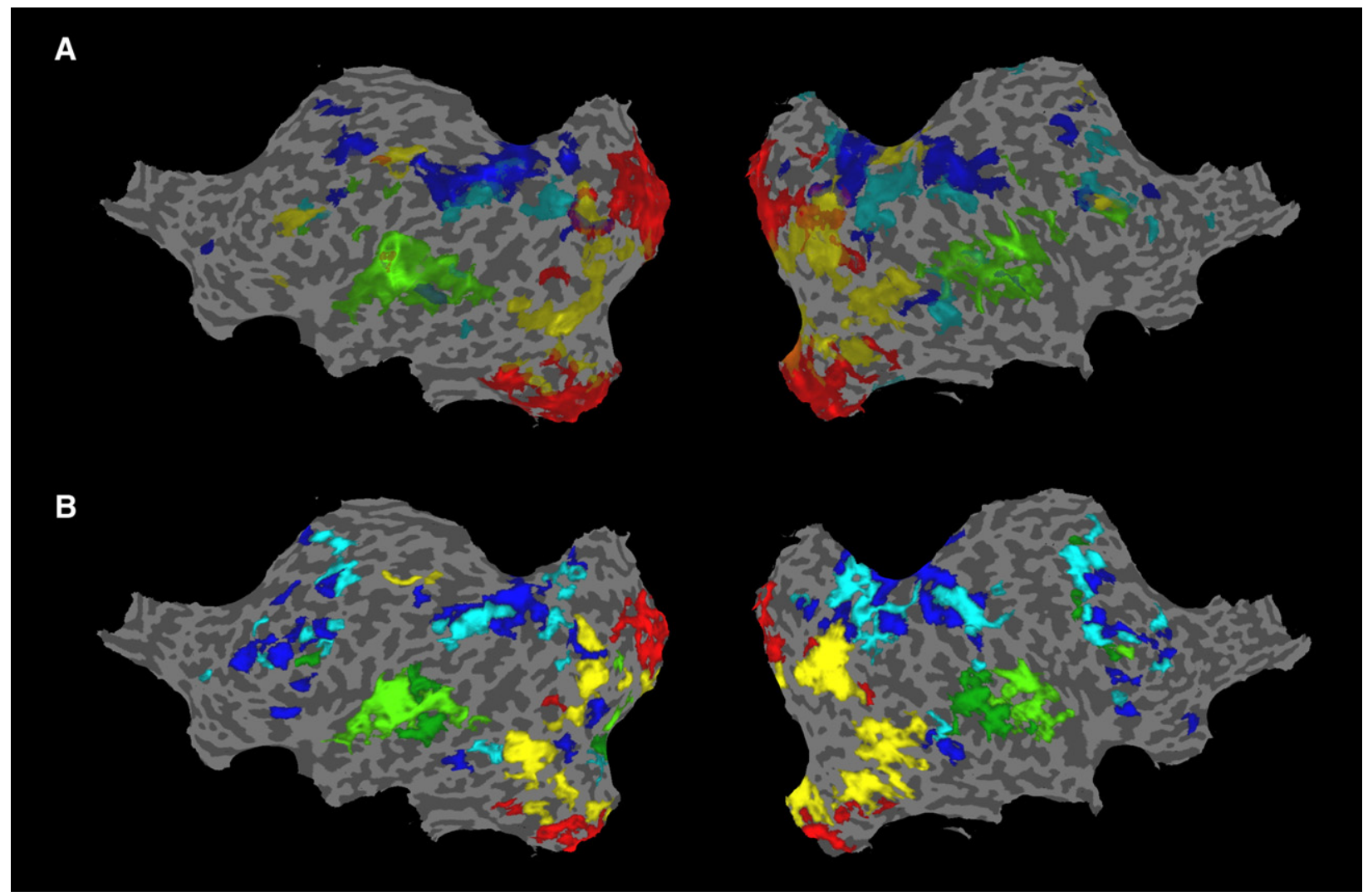

Fig. 1. Projection of maps of activation regions on inflated and flattened representation of the cortical sheet of the subject's brain ('LM', Run 1, left and right hemisphere) for ICA (A) and FCM (B): green=auditory; light blue=Imagery 1; dark blue=Imagery 2; red=visual; yellow=visual/motor. A lighter shade of a colour indicates a larger membership or IC amplitude. 

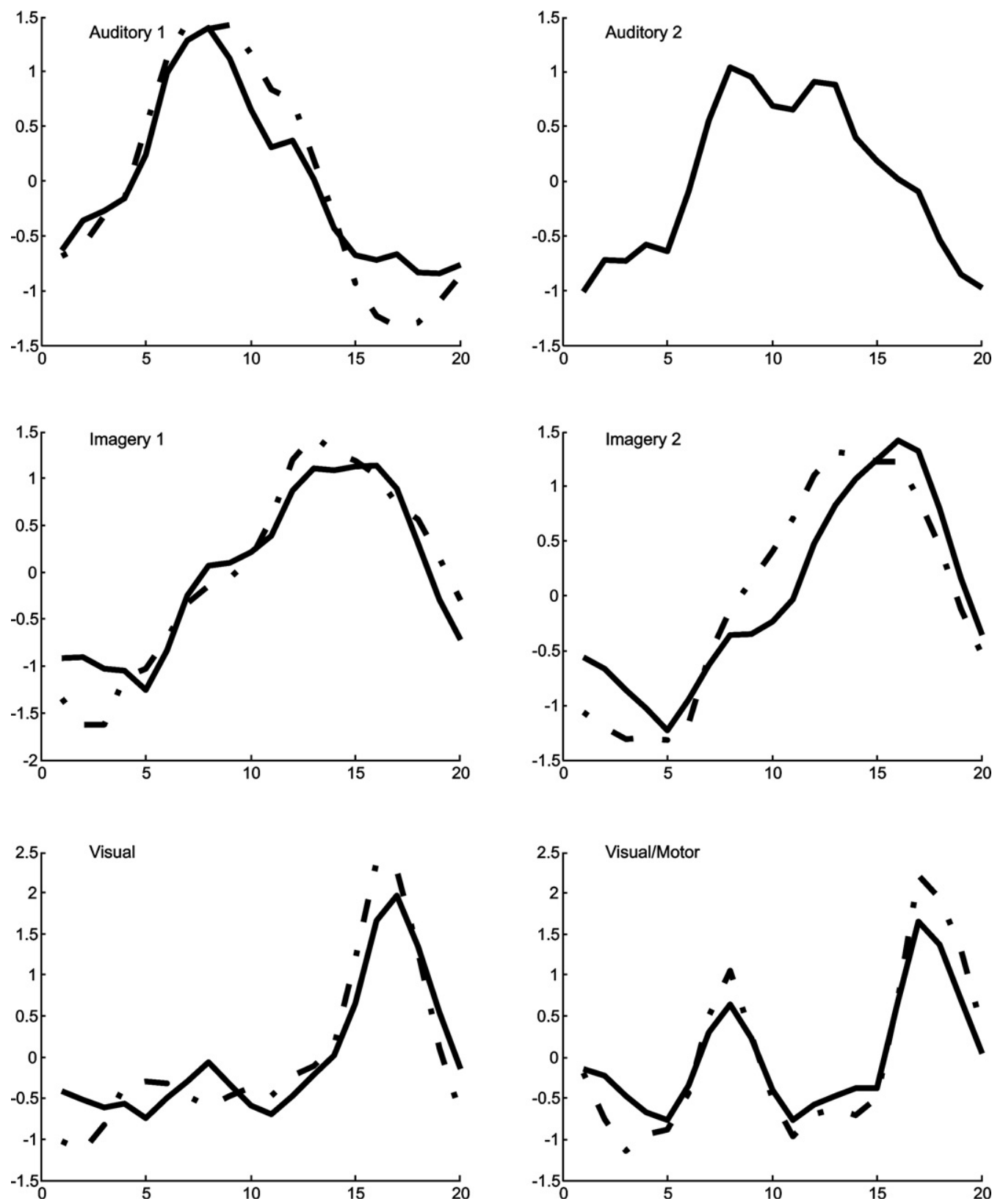

Fig. 2. Time courses of corresponding processing stages found by FCM (solid line) and ICA (dash-dot line) for subject 'LM', Run 1.

\subsection{Comparison between methods}

\subsubsection{Within- and between-subject consistency}

Table 1 illustrates ICs and clusters found by the two methods in all the data sets analysed. In all cases, both ICA and FCM were able to dissect the original time series into ICs/clusters reflecting the three main cognitive processing stages required to perform the task. The within- and between-subject consistency of these results was very high. In fact, FCM and ICA decompositions typically included an 'auditory' (10/10 with both methods), an 'imagery' (10/10 with both methods) and a 'visual/motor' IC/cluster (10/10 with ICA, 9/10 with FCM). In all cases, the spatial (anatomical) and temporal layout of these ICs/clusters closely resembled those described above for subject LM and were consistent with previous and expected results.

Table 1 also shows that, especially in the case of analysis with FCM, an even finer discrimination of neurocognitive stages could be reliably achieved. In particular, FCM was able to highlight in all the cases (10/10) a second cluster with fronto-parietal spatial distribution (labelled as 'Imagery 2 '), with a different and slightly delayed time course with respect to Imagery 1. Similarly, FCM found additional clusters presumably related to the early (auditory) and late (target related) processing stages of the tasks, respectively in 5 and 10 cases. Note that ICA decompositions of the same datasets not always produced ICs that corresponded to these additional clusters. The high within- and between-subject 
Table 1

Within- and between-subject consistency of components/clusters

\begin{tabular}{|c|c|c|c|c|c|c|c|c|}
\hline \multirow{2}{*}{$\frac{\text { Subject }}{\text { Component/cluster }}$} & \multicolumn{2}{|c|}{ All (10 runs) } & \multicolumn{2}{|c|}{$\mathrm{AB}$ (3 runs) } & \multicolumn{2}{|c|}{ CJ (4 runs) } & \multicolumn{2}{|c|}{ LM (3 runs) } \\
\hline & ICA & FCM & ICA & FCM & ICA & FCM & ICA & FCM \\
\hline Auditory 1 & 10 & 10 & 3 & 3 & 4 & 4 & 3 & 3 \\
\hline Auditory 2 & 1 & 5 & 0 & 2 & 1 & 1 & 0 & 2 \\
\hline Imagery 1 & 10 & 10 & 3 & 3 & 4 & 4 & 3 & 3 \\
\hline Imagery 2 & 5 & 10 & 1 & 3 & 1 & 4 & 3 & 3 \\
\hline Visual & 6 & 10 & 2 & 3 & 2 & 4 & 2 & 3 \\
\hline Visual/motor & 10 & 9 & 3 & 3 & 4 & 3 & 3 & 3 \\
\hline
\end{tabular}

Values in the cells indicate the number of runs in which a specific component/cluster was found.

consistency together with their anatomical layout and temporal profile suggests that this further separation is neurophysiologically meaningful and it is not due to an artificial splitting of a cluster.

\subsubsection{Spatial and temporal correspondence between ICA and FCM decompositions}

To examine the spatial and temporal similarity between ICs and clusters corresponding to the same cognitive stage we calculated, for the three main components in each data
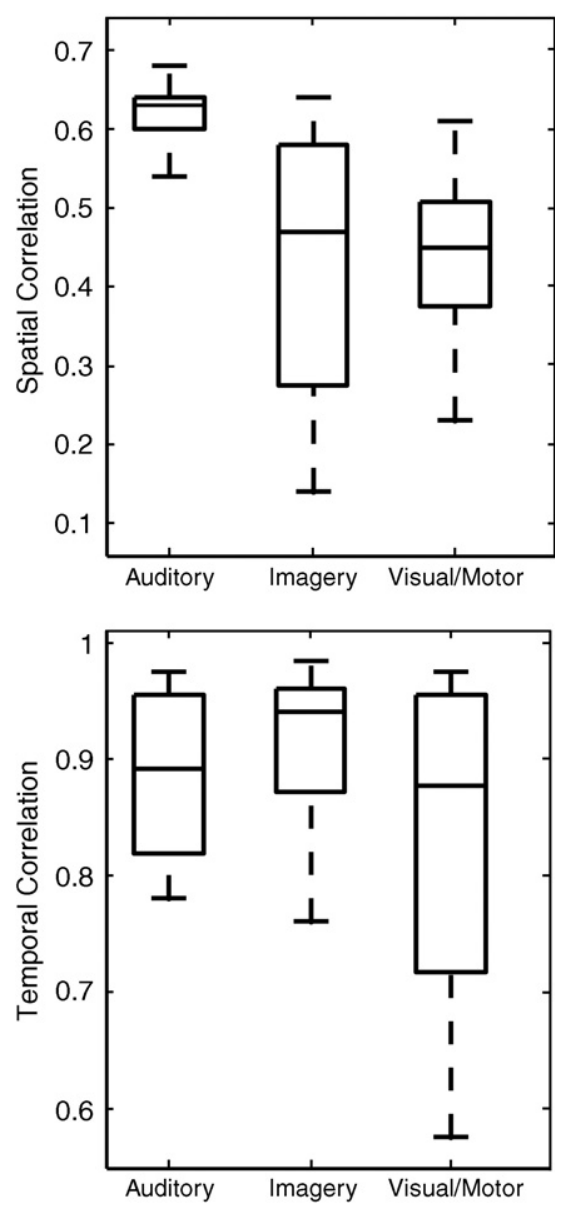

Fig. 3. Boxplot of between-methods (FCM and ICA) spatial (upper panel) and temporal (lower panel) correlation coefficient. Each boxplot has lines at the lower quartile, median and upper quartile values of computed correlations. The whiskers show the extent of the rest of the data. set, the spatial and temporal correlation coefficient of their maps and time courses. The temporal correlation coefficient was calculated between the time course of a cluster and the averaged time course of the corresponding IC. The spatial correlation coefficient was calculated without imposing any threshold on cluster membership or IC amplitude of spatial maps. Fig. 3 illustrates a summary of obtained results. Each box plot has lines at the lower quartile, median and upper quartile values of computed correlations. The whiskers show the extent of the rest of the data. Statistical significance threshold $(P<.05)$ for temporal correlation is $0.3783(T=20)$ and for spatial correlation is 0.0095 $(N \sim 30.000)$. In all cases, obtained values of correlations are above this threshold, denoting a good correspondence between the maps or time courses obtained with ICA and FCM. It is noticeable that correspondence between the maps is highest in the case of the 'auditory' IC/cluster. In the case of 'imagery' and 'visual/motor' (i.e., 'target' related) ICs/ clusters obtained median values of correlation are lower and a larger variability is present. This is mainly due to the fact that selected ICs include in some cases (five for 'imagery' and four for 'visual/motor') spatial regions which are separated into two distinct clusters in the FCM analysis.

The previous analysis of spatial correspondence between ICs and clusters was performed with unthresholded maps.

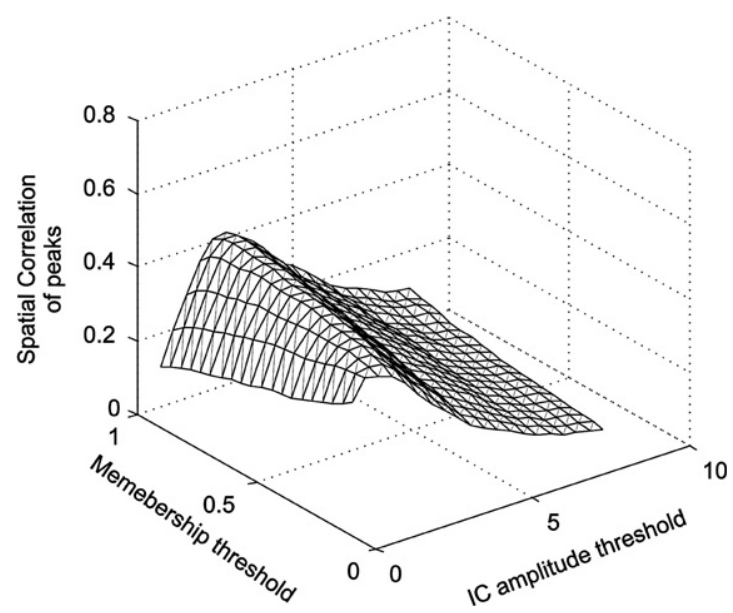

Fig. 4. Influence of thresholds (cluster membership and IC amplitude) on spatial overlap between maps. Example for subject 'LM', Run 1, spatial correlation between thresholded maps of the auditory cluster/IC found by FCM and ICA. 
Normally, however, interpretation of results is done on thresholded maps. It is thus of interest to examine how the correspondence between the peaks of ICA and FCM maps changes if cluster membership and IC-amplitude thresholds are varied. Fig. 4 shows a surface obtained by calculating the spatial cross-correlation between ICA and FCM maps after they have been thresholded at varying threshold values (i.e., values in the maps below the threshold are set to 0 ). It can be seen that the overlap of peaks is rather insensitive to changes in the cluster membership threshold, but it can be affected to a certain extent by changes in IC-amplitude threshold. Importantly, there is a relatively large range of IC amplitudes (1.5-3.5) within which there is an acceptable and constant overlap between the peaks of the two maps. Similar results were found for the other clusters.

\section{Conclusions}

In this study, we empirically compared two data-driven methods - FCM and spatial ICA - in the context of timeresolved fMRI measurements. Both FCM and spatial ICA decompositions allowed highlighting the contribution of distinct networks of brain regions to the sequential stages of a visual imagery task (auditory perception, mental imagery and behavioural response). We evaluated the maps and time courses resulting from spatial ICA and FCM in terms of their within- and between-subject consistency, which resulted to be very high in both cases. Furthermore, the calculation of spatial and temporal correlations of IC/cluster maps and time courses showed a good agreement between the results obtained with these two methods, suggesting that the partitions of the time series obtained using spatial ICA or FCM are comparable, even though they are based on substantially different principles (spatial independence vs. similarity of time courses).

The analysis of our data set also highlights a relevant distinction between the two methods and of the underlying principles. On the original (i.e., nonaveraged) time series, interesting spatio-temporal patterns of activation were correctly detected by spatial ICA but not by FCM. Conversely, when time series were averaged with respect to task onset, analyses with FCM but not spatial ICA benefited from the increase in functional contrast-to-noise of a task-related process. On averaged time series, FCM leads to a richer decomposition of the spatio-temporal patterns of activation and allowed a finer separation of the neurocognitive processes subserving the mental imagery task. In particular, processes that were united in ICA in a single component were separated into distinct and presumably meaningful clusters by FCM. Note, however, that averaging of the time series implies introducing a priori information on the stimulation paradigm (but not on the shape of the HR) and thus the subsequent analysis cannot be considered 'blind'. Also, averaging prevents examining the variability of single-trial responses and processes which are not task related (e.g., activity in the 'default mode' network). In sum, these results and considerations suggest complementary characteristics of FCM and spatial ICA decompositions, with an advantage of FCM in the analysis of task-related responses and averaged time series and an advantage of spatial ICA in the case of the variability of single trial responses and non-task-related processes are relevant.

\section{Acknowledgments}

Financial support from NWO (NWO-DFG grant DN 5519 and MaGW-VIDI grant 452-04-330) to E.F. is gratefully acknowledged.

\section{References}

[1] Ogawa S, Tank G, Menon R, Ellerman J, Kim S, Merkle H, et al. Intrinsic signal changes accompanying sensory stimulation: functional brain mapping with magnetic resonance imaging. Proc Natl Acad Sci U S A 1992;89:5951-5.

[2] Di Salle F, Formisano E, Linden DE, Goebel R, Bonavita S, Pepino A, et al. Exploring brain function with magnetic resonance imaging. Eur J Radiol 1999;30(2):84-94.

[3] Menon RS, Luknowsky DC, Gati JS. Mental chronometry using latency-resolved functional MRI. Proc Natl Acad Sci U S A 1998; 95(18):10902-7.

[4] Formisano E, Goebel R. Tracking cognitive processes with functional MRI mental chronometry. Curr Opin Neurobiol 2003;13(2):174-81.

[5] Aguirre GK, Zarahn E, D'esposito M. The variability of human, BOLD hemodynamic responses. NeuroImage 1998;8(4):360-9.

[6] Formisano E, Linden DEJ, Di Salle F, Trojano L, Esposito F, Sack AT, et al. Tracking the mind's image in the brain: I. Time-resolved fMRI during visuospatial mental imagery. Neuron 2002;35(1):185-94.

[7] Friston KJ, Holmes AP, Worsley KJ, Poline JP, Frith CD, Frackowiak RSJ. Statistical parametric maps in functional imaging: a general linear approach. Hum Brain Mapp 1994;2(4):189-210.

[8] McKeown MJ, Makeig S, Brown GG, Jung TP, Kindermann SS, Bell $\mathrm{AJ}$, et al. Analysis of fMRI data by blind separation into independent spatial components. Hum Brain Mapp 1998;6(3):160-88.

[9] Goutte C, Toft P, Rostrup E, Nielsen F, Hansen LK. On clustering fMRI time series. NeuroImage 1999;9(3):298-310.

[10] Thomas CG, Harshman RA, Menon RS. Noise reduction in BOLDbased fMRI using component analysis. NeuroImage 2002;17(3): 1521-37.

[11] McKeown MJ, Hansen LK, Sejnowsk TJ. Independent component analysis of functional MRI: what is signal and what is noise? Curr Opin Neurobiol 2003;13(5):620-9.

[12] Formisano E, Esposito F, Kriegeskorte N, Tedeschi G, Di salle F, Goebel R. Spatial independent component analysis of functional magnetic resonance imaging time-series: characterization of the cortical components. Neurocomputing 2002;49:241-54.

[13] Himberg J, Hyvärinen A, Esposito F. Validating the independent components of neuroimaging time series via clustering and visualization. NeuroImage 2004;22(3):1214-22.

[14] De Martino F., Gentile F, Esposito F, Balsi M, Di Salle F, Goebel R, et al. Classification of fMRI independent components using ICfingerprints and support vector machine classifiers. NeuroImage 2007; 34(1):177-94.

[15] Baumgartner R, Scarth G, Teichtmeister C, Somorjai R, Moser E. Fuzzy clustering of gradient-echo functional MRI in the human visual cortex: Part I. Reproducibility. J Magn Reson Imaging 1997;7(6):1094-101.

[16] Moser E, Diemling M, Baumgartner R. Fuzzy clustering of gradientecho functional MRI in the human visual cortex: Part II. Quantification. J Magn Reson Imaging 1997;7(6):1102-8.

[17] Golay X, Kollias S, Stoll G, Meier D, Valavanis A, Boesiger P. A new correlation-based fuzzy logic clustering algorithm for fMRI. Magn Reson Med 1998;40(2):249-60. 
[18] Baumgartner R, Windischberger C, Moser E. Quantification in functional magnetic resonance imaging: fuzzy clustering vs. correlation analysis. Magn Reson Med 1998;16:115-25.

[19] Baumgartner R, Ryner L, Richter W, Summers R, Jarmasz M, Somorjai R. Comparison of two exploratory data analysis methods for fMRI: fuzzy clustering vs. principal component analysis. Magn Reson Imaging 2000;18(1):89-94.

[20] Fadili MJ, Ruan S, Bloyet D, Mazoyer B. On the number of clusters and the fuzziness index for unsupervised FCA application to BOLD fMRI time series. Med Image Anal 2001;5(1):55-67.

[21] Moller U, Ligges M, Georgiewa P, Grunling C, Kaiser WA, Witte H, et al. How to avoid spurious cluster validation? A methodological investigation on simulated and fMRI data. NeuroImage 2002;17(1): $431-46$.

[22] Auffermann WF, Ngan SC, Hu X. Cluster significance testing using the bootstrap. NeuroImage 2002;17(2):583-91.

[23] Windischberger C, Barth M, Lamm C, Schroeder L, Bauer H, Gur C, et al. Fuzzy cluster analysis of high-field functional MRI data. Arti Intell Med 2003;29(3):203-23.

[24] Thirion B, Faugeras O. Feature characterization in fMRI data: the information bottleneck approach. Med Image Anal 2004;8(4): $403-19$.
[25] Goutte C, Hansen LK, Liptrot MG, Rostrup E. Feature-space clustering for fMRI meta-analysis. Hum Brain Mapp 2001;13(3):165-83.

[26] Richter W, Somorjai R, Summers R, Jarmasz M, Menon RS, Gati JS, et al. Motor area activity during mental rotation studied by timeresolved single-trial fMRI. J Cogn Neurosci 2000;12(2):310-20.

[27] Meyer-Baese A, Wismueller A, Lange O. Comparison of two exploratory data analysis methods for fMRI: unsupervised clustering versus independent component analysis. IEEE Trans Inf Technol Biomed 2004;8(3):387-98.

[28] Zadeh LA. Fuzzy sets and their application to pattern classification and clustering analysis. New York: Academic Press; 1977.

[29] Bezdek JC, Ehrlich R, Full W. FCM: the fuzzy C-means algorithm. Comput Geosci 1984;10:191-203.

[30] Mahalanobis P. On generalized distances in statistics. Proc Natl Inst Sci India 2004;12:49-55.

[31] Formisano E, Esposito F, Di Salle F, Goebel R. Cortex-based independent component analysis of fMRI time series. Magn Reson Imaging 2004;22(10):1493-504.

[32] Hyvärinen A, Oja E. A fast fixed-point algorithm for independent component analysis. Neural Comput 1997;9:1483-92.

[33] Talairach J, Tournoux P. Co-planar stereotaxic atlas of the human brain. New York: Thieme Medical Publications; 1988. 